\title{
Some Useful Functions for Functional Large Deviations.
}

\author{
Ken Duffy ${ }^{1}$ and Mark Rodgers-Lee ${ }^{2,1}$ \\ (1) Communications Network Research Institute, \\ Dublin Institute of Technology, \\ Dublin, Ireland. \\ (2) Department of Pure and Applied Mathematics, \\ Trinity College Dublin, \\ Dublin, Ireland. \\ $6^{\text {th }}$ January 2004; revised $28^{\text {th }}$ April 2004
}

\begin{abstract}
Useful descriptions of stochastic models are often provided when they are represented as functions of well understood stochastic models. Properties of the well understood model can be preserved by the representation. For example, the Large Deviation Principle (LDP) is preserved by continuous maps via the contraction principle and weak convergence is preserved by continuous maps via the continuous mapping theorem.

In a recent paper, Ganesh and O'Connell demonstrate the value of particular topological space of functions indexed by the positive real line by showing: (1) a large class of partial sums process satisfy the functional LDP in the space; and (2) the supremum map is continuous when restricted to an appropriate subspace. This makes the space useful, for example, in proving logarithmic asymptotics for the stationary waiting-time distribution at a single server queue.

This paper facilitates further deductions from the space by considering other useful maps: inversion, composition and first passage time. It is shown that composition is continuous and inversion is continuous when restricted to an appropriate subspace. First passage time is not continuous, but with additional rate-function assumptions the LDP can be deduced by explicit calculation. A number of examples of these results are presented.
\end{abstract}

*American Mathematical Society 1991 subject classifications: Primary 60F10; Secondary 91B70, 60G17.

${ }^{\dagger}$ Keywords: Functional Large Deviations, Contraction Principle, Composition, Inversion, First Passage Time. 


\section{Introduction}

Stochastic processes can often be represented by functions of more basic stochastic processes. For example, the waiting-times at a single server queue can be represented in terms of a reflected random walk and the time of ruin of an insurance company can be represented by the first passage time of a random walk.

These representations can be useful as properties of underlying stochastic process can be preserved by the function that maps to the process of interest. For example, the Large Deviation Principle (LDP) is preserved by continuous maps via the contraction principle and weak convergence is preserved by continuous maps via the continuous mapping theorem.

It has recently been shown by Ganesh and O'Connell [1] that a particular function space is useful for deducing the LDP for quantities arising in queueing systems. They show that a large class of partial sums processes satisfy the LDP and prove that the supremum map is continuous when restricted to an appropriate subspace. This makes the space particularly useful in queueing applications as the contraction principle can be used to deduce, for instance, logarithmic asymptotics for the tail of the stationary waiting-time distribution at a single server queue. For other examples of its use in queueing systems see O'Connell $[2,3]$ and Toomey [4], and for an application to Brownian motion see Hambly et al. [5].

In the spirit of Whitt $[6,7]$, this paper facilitates further deductions by considering other useful maps: inversion, composition and first passage time. It is shown that composition is continuous and inversion is continuous when restricted to an appropriate subspace. First passage time is not continuous, but with additional rate-function assumptions the LDP can be deduced by explicit calculation. A number of examples of these results are presented, including: (1) the large deviations of counting processes and their inverses, analogous to results of Puhalskii and Whitt [8]; (2) a version of Russell's random time-change [9]; and (3) a functional proof of a result of Nyrhinen $[10,11]$ regarding the LDP for the time of ruin of an insurance company.

The topological function space considered in [1] is not the only useful space over $[0, \infty)$ for the functional LDP. For example, in the space of right continuous functions having left limits equipped with various Skorohod topologies see Puhalskii [12] and Puhalskii and Whitt [8] where continuity of the supremum and inversion functions are proved and their LDP implications deduced. In [9] Russell considers the product topology and proves the continuity of composition and inversion. Dobrushin and Pechersky [13] prove the continuity of supremum in a gauge topology, for the purpose of proving logarithmic asymptotics of the waiting-time distribution at a single server queue.

The rest of this paper is organized as follows: in section 2 the function space of interest and its topology are defined; in section 3 the LDP implications of inversion's continuity are considered; in section 4 the LDP implications of composition's continuity are considered; in section 5 the LDP for first passage times discussed; all proofs are given in Appendix A. 


\section{Setup and function space motivation}

For convenience we recall the basic facts of the LDP and then introduce the function space of interest. Let $\mathcal{X}$ be a Hausdorff space with Borel $\sigma$-algebra $\mathcal{B}$ and let $\left\{\mu_{n}, n \in \mathbb{N}\right\}$ be a sequence of probability measures on $(\mathcal{X}, \mathcal{B})$. We say that $\left\{\mu_{n}, n \in \mathbb{N}\right\}$ satisfies the Large Deviation Principle (LDP) with rate-function $I: \mathcal{X} \rightarrow[0,+\infty]$ if $I$ is lower semi-continuous,

$$
-\inf _{x \in G} I(x) \leq \liminf _{n \rightarrow \infty} \frac{1}{n} \log \mu_{n}[G] \quad \text { and } \quad \limsup _{n \rightarrow \infty} \frac{1}{n} \log \mu_{n}[F] \leq-\inf _{x \in F} I(x)
$$

for all open $G$ and all closed $F$. We say that a process $\left\{X_{n}, n \in \mathbb{N}\right\}$ satisfies the LDP if $X_{n}$ is a realization of $\mu_{n}$ for each $n$. A rate-function is good if its level sets $\{x: I(x) \leq \alpha\}$ are compact for all $\alpha \geq 0$. The Contraction Principle states that if $\left\{X_{n}, n \in \mathbb{N}\right\}$ satisfies the LDP in $\mathcal{X}$ with good rate-function $I$ and $f: \mathcal{X} \rightarrow \mathcal{Y}$ is continuous, where $\mathcal{X}$ and $\mathcal{Y}$ are Hausdorff, then $\left\{f\left(X_{n}\right), n \in \mathbb{N}\right\}$ satisfies the LDP in $\mathcal{Y}$ with good rate-function given by $J(y):=\inf \{I(x): f(x)=y\}$. A proof can be found in Dembo and Zeitouni [14] Theorem 4.2.1.

Let $\mathcal{C}[0, \infty)$ denote the collection of $\mathbb{R}$ valued continuous functions on $[0, \infty)$. Let $\mathcal{A}[0, \infty)$ denote the collection of absolutely continuous functions on $[0, \infty)$ with $\phi(0)=0$. In particular, the elements of $\mathcal{A}[0, \infty)$ are exactly the integrals of functions that are elements of $\mathcal{L}^{1}[0, x)$ for all $x>0$ (for example, see Riesz and Sz.-Nagy [15]). Define the space

$$
\mathcal{Y}:=\left\{\phi \in \mathcal{C}[0, \infty): \lim _{t \rightarrow \infty} \frac{\phi(t)}{(1+t)} \text { exists in } \mathbb{R}\right\}
$$

and equip it with the topology induced by the norm

$$
\|\phi\|=\sup _{t \geq 0}\left|\frac{\phi(t)}{1+t}\right| .
$$

The space $\mathcal{Y}$ is Polish as it can be identified with the space of continuous functions on the compactified positive real line equipped with the sup norm through the bijection $\phi(t) \mapsto$ $\phi(t) /(1+t)$. Define $\mathcal{Y}_{\uparrow}:=\{\phi \in \mathcal{Y}: \phi(0)=0, \phi$ strictly increasing and $\lim \phi(t) /(1+t)>0\}$ and, for each $\mu \in \mathbb{R}, \mathcal{Y}_{\mu}:=\{\phi \in \mathcal{Y}: \lim \phi(t) /(1+t)=\mu\}$. Treat them as metric subspaces of $\mathcal{Y}$. Products of these spaces are equipped with the product topology.

The motivation for $\mathcal{Y}$ 's value in the consideration of the LDP is as follows. Given a real valued stochastic process $\left\{X_{n}, n \in \mathbb{N}\right\}$ and defining $X_{0}:=0$, the usual sample-paths of its partial sums process $\left\{\hat{S}_{n}, n \in \mathbb{N}\right\}$, defined by

$$
\hat{S}_{n}(t):=\frac{1}{n} \sum_{i=0}^{[n t]} X_{i}, \text { for } t \in[0, \infty),
$$

are not continuous functions. They are right-continuous with left-hand limits. However their polygonal approximations are continuous

$$
S_{n}(t):=\frac{1}{n} \sum_{i=0}^{[n t]} X_{i}+\left(t-\frac{[n t]}{n}\right) X_{[n t]+1}, \text { for } t \in[0, \infty) .
$$


We shall call $S_{n}$ a sample-path; it contains the same information as $\hat{S}_{n}$.

Restricting $S_{n}$ to $[0,1]$, Dembo and Zajic [16] provide general conditions under which $\left\{S_{n}, n \in \mathbb{N}\right\}$ satisfies the functional LDP with good rate-function in the space of continuous functions on $[0,1]$ equipped with the sup norm. Under their assumptions, the rate-function is given by

$$
I_{1}(\zeta)= \begin{cases}\int_{0}^{1} \lambda^{*}(\dot{\zeta}(t)) d t & \text { if } \zeta \in \mathcal{A}[0,1] \\ +\infty & \text { otherwise }\end{cases}
$$

where $\mathcal{A}[0,1]$ denotes the absolutely continuous functions on $[0,1]$ with $\phi(0)=0$ and $\lambda^{*}$ is the Legendre transform of the scaled cumulant generating function

$$
\lambda(\theta):=\lim _{n \rightarrow \infty} \frac{1}{n} \log \mathbb{E}\left[e^{n \theta S_{n}(1)}\right] .
$$

Theorem 1 of [1] establishes that if these conditions are met and $\lambda$ is differentiable at the origin, then $\left\{S_{n}, n \in \mathbb{N}\right\}$ also satisfies the functional LDP in $\mathcal{Y}$ with good rate-function

$$
I_{\infty}(\zeta)= \begin{cases}\int_{0}^{\infty} \lambda^{*}(\dot{\zeta}(t)) d t & \text { if } \zeta \in \mathcal{A}[0, \infty) \cap \mathcal{Y} \\ +\infty & \text { otherwise }\end{cases}
$$

Rate-functions of the sort in equation (3) are particularly interesting. We refer to them as of integral form with integrand $\lambda^{*}$. In general we will not assume that $\lambda^{*}$ is the Legendre transform of a scaled cumulant generating function; in particular we will not assume it is convex.

In [1] it is also proved that $f: \mathcal{Y} \rightarrow[0, \infty]$ defined by $f(\phi):=\sup _{t \geq 0} \phi(t)$ is continuous when restricted to a $\mathcal{Y}_{\mu}$ with $\mu<0$. Finally, we mention that a version of Schilder's theorem in $\mathcal{Y}$ has been proven by Deuschel and Stroock [17], and that Eichelsbacher and O'Connell [18] prove the LDP holds for partial sums of i.i.d processes in a finer topology on $\mathcal{C}[0, \infty)$.

\section{Inversion}

Define the function $g: \mathcal{Y}_{\uparrow} \rightarrow \mathcal{Y}_{\uparrow}$ by $g(\phi):=\phi^{-1}$, where $\phi^{-1}$ is the inverse of $\phi$. The motivation for the usefulness of $g$ comes from partial sums processes. Consider a process $\left\{\tau_{i}, i \in \mathbb{N}\right\}$, where $\tau_{i}$ takes values in $(0, \infty)$ for all $i$ and set $\tau_{0}:=0$. Define its sample-path process, $\left\{T_{n}, n \in \mathbb{N}\right\}$, as in equation (2); that is

$$
T_{n}(t):=\frac{1}{n} \sum_{i=0}^{[n t]} \tau_{i}+\left(t-\frac{[n t]}{n}\right) \tau_{[n t]+1}, \text { for } t \in[0, \infty) .
$$

Consider the counting process $\{N(t), t \in[0, \infty)\}$ associated with $\left\{\tau_{i}, i \in \mathbb{N}\right\}$ defined by $N(t):=\max \left\{k \geq 0: \sum_{i=0}^{k} \tau_{i} \leq t\right\}$. Construct its sample-paths by polygonal approximation to $N(n t) / n$,

$$
N_{n}(t):=\frac{1}{n} N(n t)+\left(t-\frac{1}{n} \sum_{i=0}^{N(n t)} \tau_{i}\right) \frac{1}{\tau_{N(n t)+1}}, \text { for } t \in[0, \infty) .
$$


Straightforward calculation reveals that $T_{n}\left(N_{n}(t)\right)=N_{n}\left(T_{n}(t)\right)=t$. That is, they are inversely related.

In the space of CADLAG functions the usual sample-paths of $\hat{T}_{n}$ and $\hat{N}_{n}$ are not quite inverses, which leads to added complications. In that setting, the Large Deviations implications are considered by Puhalskii and Whitt [8] in Skorohod topologies and by Russell [9] in the product topology.

Lemma 1 The function $g: \mathcal{Y}_{\uparrow} \rightarrow \mathcal{Y}_{\uparrow}$ defined by $g(\phi):=\phi^{-1}$ is continuous.

As $\phi \mapsto \phi^{-1}$ is continuous, the contraction principle can be applied and the LDP is preserved. The following theorem is analogous to Theorems 3.1 and 3.4 of [8]; see also Lemma 3.2.13 of [19].

Theorem 2 If $\left\{X_{n}, n \in \mathbb{N}\right\}$ satisfies the $L D P$ in $\mathcal{Y}_{\uparrow}$ with good rate-function $I_{\infty}(\phi)$, then $\left\{X_{n}^{-1}, n \in \mathbb{N}\right\}$ satisfies the LDP in $\mathcal{Y}_{\uparrow}$ with good rate-function $J_{\infty}(\phi)=I_{\infty}\left(\phi^{-1}\right)$. Moreover, if $I_{\infty}$ is of integral form with integrand $I$, then $J_{\infty}$ is of integral form with integrand $J(x)=$ $x I\left(x^{-1}\right)$.

Theorem 2 assumes an LDP in $\mathcal{Y}_{\uparrow}$. One way to deduce this would be by the following inclusion theorem. If $\left\{X_{n}, n \in \mathbb{N}\right\}$ satisfies the LDP in $\mathcal{Y}$ with rate-function $I_{\infty}, \mathbb{P}\left[X_{n} \in\right.$ $\left.\mathcal{Y}_{\uparrow}\right]=1$ for all $n \in \mathbb{N}$, and the effective domain of $I_{\infty}$ is a subset of $\mathcal{Y}_{\uparrow}$, then $\left\{X_{n}, n \in \mathbb{N}\right\}$ satisfies the LDP in $\mathcal{Y}_{\uparrow}$ (for example, see Lemma 4.1.5 of [14]).

Applying this theorem to an assumed LDP for the paths $\left\{T_{n}, n \in \mathbb{N}\right\}$ defined in equation (4) with good integral rate-function $I_{\infty}$ and convex integrand $I$, we get an LDP for the counting process $\left\{N_{n}, n \in \mathbb{N}\right\}$ defined in equation (5) with good integral rate-function $J_{\infty}$ and convex integrand $J(x)=x I\left(x^{-1}\right)$. To see the convexity of $J$, for $\alpha \in[0,1]$ consider $J(\alpha x+(1-\alpha) y)$, set $\eta=\alpha x /(\alpha x+(1-\alpha) y) \in[0,1]$, and use the convexity of $I$.

Care must be taken when trying to recover the one-dimensional LDP relationship for $\left\{\sum_{i=1}^{n} \tau_{i} / n, n \in \mathbb{N}\right\}$ and $\{N(n) / n, n \in \mathbb{N}\}$, first deduced by Glynn and Whitt [20]. The natural projections from $\mathcal{Y}$ to $\mathbb{R}$ are continuous. In particular $\Pi: \mathcal{Y} \rightarrow \mathbb{R}$ defined by $\Pi(\phi):=$ $\phi(1)$ is continuous, so the contraction principle can be applied. When applied to $\left\{T_{n}, n \in \mathbb{N}\right\}$, we get the LDP for $\left\{\sum_{i=1}^{n} \tau_{i} / n, n \in \mathbb{N}\right\}$ as desired, but when applied to $\left\{N_{n}, n \in \mathbb{N}\right\}$ we get an LDP for $\left\{N_{n}(1), n \in \mathbb{N}\right\}$, where

$$
N_{n}(1)=\frac{1}{n} N(n)+\left(1-\frac{1}{n} \sum_{i=0}^{N(n)} \tau_{i}\right) \frac{1}{\tau_{N(n)+1}},
$$

not $\{N(n) / n, n \in \mathbb{N}\}$. It is not difficult to check that the difference $N_{n}(1)-N(n) / n$ is in $\left[0, n^{-1}\right)$. Hence $\left\{N_{n}(1), n \in \mathbb{N}\right\}$ and $\{N(n) / n, n \in \mathbb{N}\}$ are exponentially equivalent (definition 4.2.10 of Dembo and Zeitouni [14]) and thus by Theorem 4.2.13 of [14] they satisfy the same LDP.

As the integrand $I$ is assumed convex, Jensen's inequality shows that linear paths minimize the infimum from the contraction principle and $\left\{\sum_{i=1}^{n} \tau_{i} / n, n \in \mathbb{N}\right\}$ satisfies the LDP with 
good rate-function $I$. As $J(x)=x I\left(x^{-1}\right)$ is convex, by similar arguments (and using the exponential equivalence referred to above) $\{N(n) / n, n \in \mathbb{N}\}$ satisfies the LDP with good rate-function $J$.

\section{Composition}

Define the function $h: \mathcal{Y} \times \mathcal{Y}_{\uparrow} \rightarrow \mathcal{Y}$ by $h(\phi, \psi):=\phi \circ \psi$. In the context of processes, Billingsley [21] refers to a transformation of this sort as a "random change of time". Lemma 3 shows that this function is continuous. However its application to the LDP for partial sums processes is not particularly revealing. In particular, rate-functions of integral form are not necessarily preserved by the transformation.

Russell [9] introduces a "random time-change", $h^{\prime}(\phi, \psi):=\left(\phi \circ \psi^{-1}, \psi^{-1}\right)$ which can be viewed as the composition of inversion and composition. In [9] its LDP implications are deduced in the product topology. For a large deviations motivation for composition we give an analogous result in $\mathcal{Y} \times \mathcal{Y}_{\uparrow}$ of Russell's random time-change.

Let $S_{n}$, defined in equation (2), be a sample-path of a partial sums process and $T_{n}$, defined in equation (4), be a sample-path of a timing process. Associating the time $\tau_{1}+\cdots+\tau_{i}$ to each value $X_{i}$, it is natural to assume a joint $\operatorname{LDP}$ for $\left\{\left(S_{n}, T_{n}\right), n \in \mathbb{N}\right\}$. The application of $h^{\prime}$ to $S_{n}$ and $T_{n}$, with $N_{n}=T_{n}^{-1}$, gives

$$
h^{\prime}\left(S_{n}, T_{n}\right)(t)=\left(S_{n}\left(N_{n}\right), N_{n}\right)(t)=\left(\frac{1}{n} \sum_{i=0}^{N(n t)} X_{i}+\left(t-\frac{1}{n} \sum_{i=0}^{N(n t)} \tau_{i}\right) \frac{X_{N(n t)+1}}{\tau_{N(n t)+1}}, N_{n}(t)\right) .
$$

The first argument is the sample-path of the partial sums of $X_{i}$ at the random times $\tau_{1}+$ $\cdots+\tau_{i}$.

Lemma 3 The function $h: \mathcal{Y} \times \mathcal{Y}_{\uparrow} \rightarrow \mathcal{Y}$ defined by $h(\phi, \psi):=\phi \circ \psi$ is continuous.

As $(\phi, \psi) \mapsto\left(\phi, \psi^{-1}\right) \mapsto\left(\phi \circ \psi^{-1}, \psi^{-1}\right)$ is continuous, the contraction principle can be applied to deduce the following version of Russell's random time-change (see Theorem 5.1, Lemma 5.2 of [9]). It is in [9] that the rate-function relationship $J(x, y)=y I(x / y, 1 / y)$ first appears.

Theorem 4 If $\left\{\left(X_{n}, Y_{n}\right), n \in \mathbb{N}\right\}$ satisfies the $L D P$ in $\mathcal{Y} \times \mathcal{Y}_{\uparrow}$ with good rate-function $I_{\infty}(\phi, \psi)$, then $\left\{\left(X_{n} \circ Y_{n}^{-1}, Y_{n}^{-1}\right), n \in \mathbb{N}\right\}$ satisfies the LDP in $\mathcal{Y} \times \mathcal{Y}_{\uparrow}$ with good rate-function $J_{\infty}(\zeta, \xi)=$ $I_{\infty}\left(\zeta \circ \xi^{-1}, \xi^{-1}\right)$. Moreover, if $I_{\infty}$ is of integral form with integrand $I$, then $J_{\infty}$ is of integral form with integrand $J(x, y)=y I(x / y, 1 / y)$.

Applying this theorem to an assumed joint LDP for the paths $\left\{\left(S_{n}, T_{n}\right), n \in \mathbb{N}\right\}$ defined in equations (2) and (4) with good integral rate-function $I_{\infty}$ and jointly convex integrand $I(x, y)$, we get an LDP for the random time-changed process $\left\{\left(S_{n} \circ N_{n}, N_{n}\right), n \in \mathbb{N}\right\}$ defined in equation (6) with good integral rate-function $J_{\infty}$ and convex integrand $J(x, y)=y I(x / y, 1 / y)$. 
To see the convexity of $J(x, y)$, set $\vec{x}=\left(x_{1}, x_{2}\right)$ and $\vec{y}=\left(y_{1}, y_{2}\right)$, let $\alpha \in[0,1]$, note that

$$
J(\alpha \vec{x}+(1-\alpha) \vec{y})=\frac{1}{\alpha x_{2}+(1-\alpha) y_{2}} I\left(\eta\left(\frac{x_{1}}{x_{2}}, \frac{1}{x_{2}}\right)+(1-\eta)\left(\frac{y_{1}}{y_{2}}, \frac{1}{y_{2}}\right)\right),
$$

where $\eta=\alpha x_{2} /\left(\alpha x_{2}+(1-\alpha) y_{2}\right)$, and use the convexity of $I$.

As with inversion, care is needed when considering the one-dimensional projection. When applied to $\left\{\left(S_{n}, T_{n}\right), n \in \mathbb{N}\right\}$, we get the LDP in $\mathbb{R} \times(0, \infty)$ for $\left\{\left(\sum_{i=1}^{n} X_{i} / n, \sum_{i=1}^{n} \tau_{i} / n\right), n \in\right.$ $\mathbb{N}\}$ with rate-function $I(x, y)$, as desired. However, the one-dimensional projection of $\left(S_{n} \circ\right.$ $\left.N_{n}, N_{n}\right)$ gives for its first argument:

$$
\left(S_{n} \circ N_{n}\right)(1)=\frac{1}{n} \sum_{i=0}^{N(n)} X_{i}+\left(t-\frac{1}{n} \sum_{i=0}^{N(n)} \tau_{i}\right) \frac{X_{N(n)+1}}{\tau_{N(n)+1}} .
$$

Without further assumption $\left\{S_{n} \circ N_{n}(1), n \in \mathbb{N}\right\}$ is not exponentially equivalent to the process we are interested in $\left\{n^{-1} \sum_{i=0}^{N(n)} X_{i}, n \in \mathbb{N}\right\}$, as for each $n$ their difference is in $\left[0, X_{N(n)+1} / n\right)$. In other words, we need an assumption regarding the tail of $X_{i}$, for all $i$, to ensure exponential equivalence. Dembo and Zajic [16] introduce such conditions to move from an LDP for polygonal approximations $\left\{S_{n}, n \in \mathbb{N}\right\}$ to CADLAG functions $\left\{\hat{S}_{n}, n \in \mathbb{N}\right\}$.

Using Jensen's inequality and the joint convexity of $J,\left\{\left(S_{n}\left(N_{n}\right), N_{n}\right)(1), n \in \mathbb{N}\right\}$ satisfies the LDP with rate-function $J$. Furthermore, the projection $\left(S_{n}\left(N_{n}(1)\right), N_{n}(1)\right) \mapsto S_{n}\left(N_{n}(1)\right)$ gives the LDP for $\left\{S_{n}\left(N_{n}(1)\right), n \in \mathbb{N}\right\}$ (defined in equation (7)) with good, convex ratefunction $J^{\prime}(x)=\inf _{z} J(x, z)$. The convexity of $J^{\prime}(x)$ follows noting that $\inf _{z} J(\alpha x+(1-$ $\alpha) y, z)=\inf _{z_{1}, z_{2}} J\left(\alpha x+(1-\alpha) y, \alpha z_{1}+(1-\alpha) z_{2}\right)$ and using the joint convexity of $J(x, z)$.

\section{First passage time}

Consider a partial sums process $\left\{\sum_{i=1}^{n} X_{i}, n \in \mathbb{N}\right\}$. We are interested in the first time it is greater than $n u$, some $u>0$,

$$
\tau(n u):=\inf \left\{m \in \mathbb{N}: \sum_{i=1}^{m} X_{i}>n u\right\},
$$

where the infimum over the empty set is defined to be $+\infty$. Define the function $\tau_{u}: \mathcal{Y} \rightarrow[0, \infty]$ by $\tau_{u}(\phi):=\inf \{t: \phi(t)>u\}$ and equip $[0, \infty]$ with the the Borel topology on the extended real line restricted to $[0, \infty]$. The value $\tau_{u}(\phi)$ is the first time $\phi$ passes out of the region $(-\infty, u]$. Consider its application to $S_{n}$ defined in equation (2),

$$
\tau_{u}\left(S_{n}\right)=\inf \left\{t: \frac{1}{n} \sum_{i=1}^{[n t]} X_{i}+\left(t-\frac{[n t]}{n}\right) X_{[n t]+1}>u\right\} .
$$


It is not quite the functional equivalent of $\tau(n u) / n$, but as $\tau(n u) / n-\tau_{u}\left(S_{n}\right)$ is in $[0,1 / n)$, $\{\tau(n u) / n, n \in \mathbb{N}\}$ and $\left\{\tau_{u}\left(S_{n}\right), n \in \mathbb{N}\right\}$ are exponentially equivalent.

However $\tau_{u}$ is not continuous, so the contraction principle cannot be applied. For example, consider the sequence of $\mathcal{Y}$ functions

$$
\eta_{n}(t):= \begin{cases}t\left(u+n^{-1}\right) & \text { if } t \in[0,1] \\ u+n^{-1} & \text { if } t \in(1, \infty)\end{cases}
$$

for $n \in \mathbb{N}$. The discontinuity arises as $\lim _{n \rightarrow \infty} \tau_{u}\left(\eta_{n}\right)=1$, but $\tau_{u}\left(\lim _{n \rightarrow \infty} \eta_{n}\right)=+\infty$.

As we cannot use the contraction principle to deduce the LDP, we need another approach. Our approach is explicit calculation under the assumption of an integral rate-function with convex integrand $I$. We also assume a stability condition: the existence of unique $\chi \neq 0$ such that $I(\chi)=0$. If $I(0)=0$, the induced measures are not necessarily exponentially tight. For a prototype of this argument in a setting where exponential tightness is automatic, see Duffy and Dukes [22].

Theorem 5 If $\left\{X_{n}, n \in \mathbb{N}\right\}$ satisfies the LDP in $\mathcal{Y}$ with good integral rate-function $I_{\infty}$ and convex integrand $I$ with unique $\chi \neq 0$ such that $I(\chi)=0$, then $\left\{\tau_{u}\left(X_{n}\right), n \in \mathbb{N}\right\}$ satisfies the LDP in $[0, \infty]$ with good rate-function

$$
J(x)= \begin{cases}\lim _{z \rightarrow 0} z I\left(u z^{-1}\right) & \text { if } x=0, \\ x I\left(u x^{-1}\right) & \text { if } x \in(0, \infty), \\ +\infty & \text { if } x=+\infty \text { and } \chi>0, \\ 0 & \text { if } x=+\infty \text { and } \chi<0 .\end{cases}
$$

Consider an insurance company with initial capital $u$. Let the variable $X_{n}$ represent the difference in premiums acquired and the value of claims made in accounting period number $n$. We are interested in the scaled time of ruin: the first time the accumulated claims are greater than the premium accrued plus the scaled initial capital, that is $\tau(n u) / n$.

Assume that the sequence $\left\{S_{n}, n \in \mathbb{N}\right\}$ (defined in equation (2)), the sample-paths of the difference between premium accrued and value of claims made, satisfies the conditions of Theorem 5. Using the exponential equivalence described at the start of this section, an LDP for the time of ruin, $\{\tau(n u) / n, n \in \mathbb{N}\}$, is deduced with rate-function defined in equation (8). This provides an alternative means of proving an important result of Nyrhinen [10,11].

\section{Acknowledgments:}

This work was supported by Science Foundation Ireland under the National Development Plan. The authors wish to thank Wayne Sullivan for helpful discussions. They also wish to thank Charles Pfister, Anatolii Puhalskii and Raymond Russell for useful comments on an earlier draft that shortened many of the proofs. 


\section{A Proofs}

Proof: Lemma 1, continuity of inversion.

We must first show that if $\psi \in \mathcal{Y}_{\uparrow}$, then $\psi^{-1} \in \mathcal{Y}_{\uparrow}$. As $\psi^{-1}$ is strictly increasing, if $\psi \in \mathcal{Y}_{\uparrow}$ with $\psi(t) /(1+t) \rightarrow \mu, \lim \psi^{-1}(t) /(1+t)=\lim \psi^{-1}(\psi(t)) /(1+\psi(t))=1 / \mu$. As $\psi$ is continuous and strictly increasing, $\psi^{-1}$ is continuous and strictly increasing.

To check continuity of $\psi \rightarrow \psi^{-1}$, as $\mathcal{Y}_{\uparrow}$ is a metric space it suffices to show that $\psi_{n} \rightarrow \psi$ implies $\psi_{n}^{-1} \rightarrow \psi^{-1}$. By assumption, $\lim _{t \rightarrow \infty} \psi(t) /(1+t)=\mu$, for some $\mu>0$. Hence, given $\mu>\delta>0$, there exists finite $T_{0}$ such that

$$
\left|\frac{\psi(t)}{1+t}-\mu\right|<\frac{\delta}{2}, \text { for all } t>T_{0} .
$$

As $\psi_{n} \rightarrow \psi$, there exists $N_{\delta} \in \mathbb{N}$ such that

$$
\left|\frac{\psi_{n}(t)}{1+t}-\frac{\psi(t)}{1+t}\right|<\frac{\delta}{2} \text { for all } t \geq 0 \text { and } n>N_{\delta}
$$

Thus, by the triangle inequality,

$$
\left|\psi_{n}(t)-\mu(1+t)\right|<\delta(1+t) \text { for all } t>T_{0} \text { and } n>N_{\delta} .
$$

We wish to use these bounds on $\psi(s), s>T_{0}$, to deduce bounds on $\psi^{-1}(s)$. Defining $T_{\delta}:=T_{0}+2 \delta\left(1+T_{0}\right) /(\mu-\delta)$, straightforward geometry reveals

$$
\left|\psi_{n}^{-1}(s)-\psi^{-1}(s)\right|<\frac{2 \delta s}{(\mu-\delta)^{2}}, \text { for all } s>\psi\left(T_{\delta}\right) \text { and } n>N_{\delta} .
$$

For $\delta \in[0, \mu), 2 \delta /(\mu-\delta)^{2}$ is a continuous, strictly increasing function onto $[0, \infty)$. Choosing $\delta$ such that $2 \delta /(\mu-\delta)^{2}=\epsilon$, we have

$$
\left|\psi_{n}^{-1}(s)-\psi^{-1}(s)\right|<\epsilon s \leq \epsilon(1+s) \text {, for all } s \in\left(\psi\left(T_{\delta}\right), \infty\right) \text { and } n \geq N_{\delta} .
$$

To complete the continuity argument we must calculate a bound on the compact interval $\left[0, \psi\left(T_{\delta}\right)\right]$. For $t \geq 0$, define

$$
f_{1}(t):=\psi(t+\epsilon) \text { and } f_{2}(t):= \begin{cases}\psi(t-\epsilon) & \text { for all } t \geq \epsilon, \\ 0 & \text { otherwise }\end{cases}
$$

and note that $f_{1}^{-1}(s)=\psi^{-1}(s)-\epsilon$ for all $s \geq 0$ and $f_{2}^{-1}(s)=\psi^{-1}(s)+\epsilon$ for all $s \in[\psi(\epsilon), \infty)$. Set

$$
d:=\min \left\{\inf _{t \in[0, T-\epsilon]}\left|f_{1}(t)-\psi(t)\right|, \inf _{t \in[\epsilon, T+\epsilon]}\left|f_{2}(t)-\psi(t)\right|\right\} ;
$$

it is strictly positive. To see this, note that $F_{1}(t)=\left|f_{1}(t)-\psi(t)\right|$ and $F_{2}(t)=\left|f_{2}(t)-\psi(t)\right|$ are continuous functions on the compact intervals $\left[0, T_{\delta}-\epsilon\right]$ and $\left[\epsilon, T_{\delta}+\epsilon\right]$. Thus both infima 
in equation (10) are achieved at, say, $t_{1}, t_{2}$ respectively. If $d=0$, then either $\psi\left(t_{1}+\epsilon\right)=\psi\left(t_{1}\right)$ or $\psi\left(t_{2}-\epsilon\right)=\psi\left(t_{2}\right)$. This is not possible as $\psi$ is strictly increasing.

As $d>0$, there exists $N_{d}$ such that $\left|\psi_{n}(t)-\psi(t)\right|<d$ for all $t \in\left[0, T_{\delta}+\epsilon\right]$ and $n \geq N_{d}$. Straightforward geometry reveals that

$$
\left|\psi_{n}^{-1}(s)-\psi^{-1}(s)\right|<\epsilon \leq \epsilon(1+s) \text {, for all } s \in\left[0, \psi\left(T_{\delta}\right)\right] \text { and } n \geq N_{d} .
$$

Given $\epsilon>0$, set $N_{\epsilon}=\max \left\{N_{\delta}, N_{d}\right\}$, then combining equations (9) and (11),

$$
\left|\frac{\psi_{n}^{-1}(s)}{1+s}-\frac{\psi^{-1}(s)}{1+s}\right|<\epsilon \text { for all } s \in[0, \infty) \text { and } n \geq N_{\epsilon},
$$

so that $\psi \rightarrow \psi^{-1}$ is continuous.

Proof: Theorem 2, LDP from inversion. For the part regarding integral rate-functions, we must first show that if $\psi \in \mathcal{A}[0, \infty) \cap \mathcal{Y}_{\uparrow}$, then $\psi^{-1} \in \mathcal{A}[0, \infty) \cap \mathcal{Y}_{\uparrow}$. Since $\psi \in \mathcal{A}[0, \infty)$ there exists $f$ such that $\psi(t)=\int_{0}^{t} f(x) d x$, where $f \in \mathcal{L}^{1}[0, x)$ for all $x>0$ and $d x$ denotes Lebesgue measure. Define a signed measure $\mu$ on $\mathcal{B}(\mathbb{R})$ by $\mu[B]:=\int_{B} f(x) d x$. Since $\psi$ is a strictly increasing continuous function on $[0, \infty), \mu(a, b]=\int_{a}^{b} f(x) d x \geq 0$ for all $a<b \in[0, \infty)$, so by Carathéodry's extension theorem $\mu[B] \geq 0$ for all $B \in \mathcal{B}(\mathbb{R})$. Consequently the set $\{x: f(x)<0\}$ has zero measure, since otherwise $\int_{\{x: f(x)<0\}} f(x) d x<0$. We may therefore assume, without loss of generality, that $f \geq 0$.

Defining $D=\{x \in[0, \infty): f(x)=0\}, \mu[D]=\int_{D} f(x) d x=0$. Hence

$$
\mu[B]=\int_{B \cap D^{c}} f(x) d x \quad \forall B \in \mathcal{B}(\mathbb{R}) .
$$

Since $\psi^{-1}$ is strictly increasing it follows that

$$
\mu \circ \psi^{-1}(a, b]=\mu\left[\psi^{-1}(a, b]\right]=\int_{\psi^{-1}(a)}^{\psi^{-1}(b)} f(y) d y=\psi\left(\psi^{-1}(b)\right)-\psi\left(\psi^{-1}(a)\right)=b-a,
$$

and so $\mu \circ \psi^{-1}$ is equal to Lebesgue measure. Define

$$
g(y):= \begin{cases}1 & \text { (arbitrary) if } y \in \psi(D) \\ \frac{1}{f \circ \psi^{-1}} & \text { otherwise. }\end{cases}
$$

Then by Theorem C, page 163 of Halmos [23], we have

$$
\begin{aligned}
\int_{0}^{d} g(y) d y & =\int_{0}^{d} g(y) d \mu \circ \psi^{-1}(y)=\int_{0}^{\psi^{-1}(d)} g(\psi(x)) d \mu(x) \\
& =\int_{0}^{\psi^{-1}(d)} \frac{1}{f \circ \psi^{-1}(\psi(x))} f(x) d x=\int_{0}^{\psi^{-1}(d)} d x=\psi^{-1}(d) .
\end{aligned}
$$


and $\psi^{-1} \in \mathcal{A}[0, \infty)$ with integrand $g$.

That $J_{\infty}$ is of integral form then follows using the contraction principle and the change of variable formula (see the proof of Theorem $3.4[8]$ for details).

Proof: Lemma 3, continuity of composition. The function $\phi \circ \psi$ is in $\mathcal{Y}$, as

$$
\frac{\phi(\psi(t))}{1+t}=\frac{\phi(\psi(t))}{1+\psi(t)} \frac{1+\psi(t)}{1+t} .
$$

Let $\lim _{t \rightarrow \infty} \phi(t) /(1+t)=\mu, \lim _{t \rightarrow \infty} \psi(t) /(1+t)=\nu>0$. Given $\epsilon \in(0,4)$, choose $N_{\epsilon}$ such that for all $n \geq N_{\epsilon}$ and all $t \geq 0$,

$$
\left|\frac{\phi(t)}{1+t}-\frac{\phi_{n}(t)}{1+t}\right| \leq \frac{\epsilon}{4} \quad \text { and } \quad\left|\frac{\psi(t)}{1+t}-\frac{\psi_{n}(t)}{1+t}\right| \leq \frac{\epsilon}{4}
$$

Choose $K_{\phi}$ such that for all $t \geq K_{\phi}$,

$$
\left|\frac{\phi(t)}{1+t}-\mu\right| \leq \frac{\epsilon}{4}
$$

This implies that for all $n \geq N_{\epsilon}$ and all $s, t \geq K_{\phi}$,

$$
\left|\frac{\phi_{n}(t)}{1+t}-\mu\right| \leq \frac{\epsilon}{2} \quad \text { and } \quad\left|\frac{\phi(s)}{1+s}-\frac{\phi_{n}(t)}{1+t}\right| \leq \frac{3 \epsilon}{4}<\epsilon .
$$

We can also choose $K_{\psi}$ such that for all $t \geq K_{\psi}$ and $n \geq N_{\epsilon}, \psi(t) \geq K_{\phi}$ and $\psi_{n}(t) \geq K_{\phi}$. Therefore, if $t \geq K_{\psi}$ and $n \geq N_{\epsilon}$, using the elementary identity $2(a b-c d)=(a-c)(b-d)+$ $(a+c)(b-d)$,

$$
\left|\frac{\phi(\psi(t))}{1+t}-\frac{\phi_{n}\left(\psi_{n}(t)\right)}{1+t}\right|=\left|\frac{1+\psi(t)}{1+t} \frac{\phi(\psi(t))}{1+\psi(t)}-\frac{1+\psi_{n}(t)}{1+t} \frac{\phi_{n}\left(\psi_{n}(t)\right)}{1+\psi_{n}(t)}\right| \leq \epsilon(\|\psi\|+1) .
$$

For $t \leq K_{\psi}$, there exists some $C<\infty$ such that $\psi\left(K_{\psi}\right) \leq C$ and $\psi_{n}\left(K_{\psi}\right) \leq C$, for all $n \geq N_{\epsilon}$. On $[0, C], \phi$ is uniformly continuous and therefore there exits $\delta_{\epsilon}$ such that

$$
|\phi(s)-\phi(t)| \leq \frac{\epsilon}{2} \quad \text { whenever } \quad|s-t| \leq \delta_{\epsilon} .
$$

Choose $N_{\epsilon}^{\prime} \geq N_{\epsilon}$ such that for all $n \geq N_{\epsilon}^{\prime}$

$$
\sup _{t \in\left[0, K_{\psi}\right]}\left|\psi_{n}(t)-\psi(t)\right| \leq \delta_{\epsilon} \quad \text { and } \quad \sup _{s \in[0, C]}\left|\phi(s)-\phi_{n}(s)\right| \leq \frac{\epsilon}{2} .
$$

Then, if $t \leq K_{\psi}$ and $n \geq N_{\epsilon}^{\prime}$,

$$
\left|\phi(\psi(t))-\phi_{n}\left(\psi_{n}(t)\right)\right| \leq\left|\phi(\psi(t))-\phi\left(\psi_{n}(t)\right)\right|+\left|\phi\left(\psi_{n}(t)\right)-\phi_{n}\left(\psi_{n}(t)\right)\right| \leq \epsilon .
$$

The proof follows combining equations (12) and (13). 
Proof: Theorem 4, LDP from composition. Consider rate-functions of integral form. If $\phi, \psi \in \mathcal{A}[0, \infty)$ and $\psi$ is strictly increasing, that $\phi \circ \psi \in \mathcal{A}[0, \infty)$ follows from the final Theorem on page 55 of Riesz and Sz.-Nagy [15]. Thus if $\eta \in \mathcal{A}[0, \infty) \cap \mathcal{Y}$, with $\phi \in \mathcal{A}[0, \infty) \cap \mathcal{Y}$ and $\psi \in \mathcal{A}[0, \infty) \cap \mathcal{Y}_{\uparrow}$,

$$
\begin{aligned}
J_{\infty}(\eta, \xi) & =\inf \left\{I_{\infty}(\phi, \psi):\left(\phi \circ \psi^{-1}, \psi^{-1}\right)=(\eta, \xi)\right\} \\
& =I_{\infty}\left(\eta \circ \xi^{-1}, \xi^{-1}\right) \\
& =\int_{0}^{\infty} I\left(\eta\left(\xi^{-1}(s)\right), \dot{\xi}^{-1}(s)\right) d s \\
& \left.=\int_{0}^{\infty} I(\dot{\eta}(s) / \dot{\xi}(s), 1 / \dot{\xi}(s)) \dot{\xi}(s) d s\right\}, \\
& =\int_{0}^{\infty} J(\dot{\eta}(s), \dot{\xi}(s)) d s,
\end{aligned}
$$

where the second last equality comes changing variable $s \mapsto \xi(s)$ and $J(x, y):=y I(x / y, 1 / y)$.

PRoOF: Theorem 5, LDP for first passage time.

Let $G_{\epsilon}(x)$ denote a decreasing sequence of open sets as $\epsilon \rightarrow 0$ around $x$ in $[0, \infty]$. If $x \in[0, \infty)$ take $G_{\epsilon}(x)=B_{\epsilon}(x)$, the open ball of radius $\epsilon$ around $x$. If $x=\infty$, take $G_{\epsilon}=$ $(1 / \epsilon,+\infty]$. If we can establish

$$
\lim _{\epsilon \rightarrow 0} \liminf _{n \rightarrow \infty} \frac{1}{n} \log \mathbb{P}\left[\tau_{u}\left(X_{n}\right) \in G_{\epsilon}(x)\right]=\lim _{\epsilon \rightarrow 0} \limsup _{n \rightarrow \infty} \frac{1}{n} \log \mathbb{P}\left[\tau_{u}\left(X_{n}\right) \in G_{\epsilon}(x)\right],
$$

for all $x \in[0, \infty]$, then we can deduce bounds similar to those for the LDP in equation (1), but with the upper bound for all compact sets, $K$, instead of all closed sets $F$ (Theorem 4.1.11 of [14]). To deduce the upper bound for all closed $F$, one then proves the measures are exponentially tight. That is, for each $\alpha>0$, there exists a compact set $K_{\alpha}$ such that its complement $K_{\alpha}^{c}$ satisfies $\lim \sup n^{-1} \log \mu_{n}\left[K_{\alpha}^{c}\right]<-\alpha$. A consequence of this approach is that the rate-function is automatically good (Lemma 1.2.18 of [14]).

First we prove equation (14). For the upper bound note that given $x \in(0, \infty)$ and $0<\epsilon<x$,

$$
\mathbb{P}\left[\tau_{u}\left(X_{n}\right) \in B_{\epsilon}(x)\right] \leq \mathbb{P}\left[X_{n} \in\left\{\phi: \sup _{s \in[0, x-\epsilon]} \phi(s) \leq u\right\} \cap\left\{\phi: \sup _{s \in[x-\epsilon, x+\epsilon]} \phi(s) \geq u\right\}\right] .
$$

As the supremum of a continuous function is attained on a compact interval, the final two sets are closed in $\mathcal{Y}$ and the functional LDP upper-bound can be applied

$$
\limsup _{n \rightarrow \infty} \frac{1}{n} \log \mathbb{P}\left[\tau_{u}\left(X_{n}\right) \in B_{\epsilon}(x)\right] \leq-\inf \left\{I_{\infty}(\phi): \sup _{s \in[0, x-\epsilon]} \phi(s) \leq u, \sup _{s \in[x-\epsilon, x+\epsilon]} \phi(s) \geq u\right\} .
$$

Jensen's inequality and the convexity of $I$ give

$$
\lim _{\epsilon \rightarrow 0} \inf \left\{I_{\infty}(\phi): \sup _{s \in[0, x-\epsilon]} \phi(s) \leq u, \sup _{s \in[x-\epsilon, x+\epsilon]} \phi(s) \geq u\right\} \geq \lim _{\epsilon \rightarrow 0} \inf _{s \in[x-\epsilon, x+\epsilon]} s I\left(\frac{u}{s}\right)=x I\left(\frac{u}{x}\right),
$$


where the last equality comes because the strict convexity of $I(x)$ implies $x I\left(u x^{-1}\right)$ is strictly convex for $x>0$.

For the lower bound, let $\chi \neq 0$ be such that $I(\chi)=0$ and define the function

$$
\psi(t):= \begin{cases}t u / x & \text { for } t \in[0, x+\epsilon] \\ (x+\epsilon) u / x+\chi(t-(x+\epsilon)) & \text { for all } t \in[x+\epsilon, \infty)\end{cases}
$$

The function $\psi$ is our candidate for the most likely path. For $0<\epsilon<x$, set $\delta=u \epsilon /\left(x+x^{2}+x \epsilon\right)$ and for the lower bound note that

$$
\mathbb{P}\left[\tau_{u}\left(X_{n}\right) \in B_{\epsilon}(x)\right] \geq \mathbb{P}\left[X_{n} \in\left\{\phi \in B_{\delta}(\psi)\right\}\right],
$$

where $B_{\delta}(\psi)$ is the open ball of radius $\delta$ around $\psi$ in $\mathcal{Y}$. Applying the functional lower bound and noting that $\epsilon \rightarrow 0$ implies $\delta \rightarrow 0$,

$$
\lim _{\epsilon \rightarrow 0} \liminf _{n \rightarrow \infty} \frac{1}{n} \log \mathbb{P}\left[\tau_{u}\left(X_{n}\right) \in B_{\epsilon}(x)\right] \geq-\lim _{\epsilon \rightarrow 0} \inf _{s \in(x-\epsilon, x+\epsilon)} s I\left(\frac{u}{s}\right)=-x I\left(\frac{u}{x}\right) .
$$

If $x=0$, then $[0, \epsilon)$ is a decreasing sequence of sets around 0 . Similar arguments hold to those for $x \in(0, \infty)$ and as $X_{n}(0)=0$, the limit equals $\lim _{z \rightarrow 0} z I(u / z)$. If $x=\infty$, then $(1 / \epsilon, \infty]$ is a decreasing sequence of open sets around $+\infty$. There are two cases: if $\chi>0$, then for any $\epsilon>0$

$$
\mathbb{P}\left[\tau_{u}\left(X_{n}\right) \in(1 / \epsilon, \infty]\right] \leq \mathbb{P}\left[X_{n} \in\left\{\phi: \sup _{s \in[0,1 / \epsilon]} \phi(s) \leq u\right\}\right]
$$

and therefore as $I(0)>0$,

$$
\lim _{\epsilon \rightarrow 0} \limsup _{n \rightarrow \infty} \frac{1}{n} \log \mathbb{P}\left[\tau_{u}\left(X_{n}\right) \in(1 / \epsilon, \infty]\right] \leq-\lim _{\epsilon \rightarrow 0} \inf _{s \in[1 / \epsilon, \infty]} s I\left(\frac{u}{s}\right)=-\infty .
$$

With $\chi<0$ and $x=+\infty$, set $\hat{\phi}(t)=\chi t$ and $0<\delta<-m$. If $\phi \in \mathcal{B}_{\delta}(\hat{\phi})$, then $\tau_{u}(\phi)=+\infty$. Hence for all $\epsilon>0$,

$$
\mathbb{P}\left[\tau_{u}\left(X_{n}\right) \in(1 / \epsilon, \infty]\right] \geq \mathbb{P}\left[X_{n} \in B_{\delta}(\hat{\phi})\right] .
$$

As $I_{\infty}(\hat{\phi})=0$,

$$
\lim _{\epsilon \rightarrow 0} \liminf _{n \rightarrow \infty} \frac{1}{n} \log \mathbb{P}\left[\tau_{u}\left(X_{n}\right) \in(1 / \epsilon, \infty]\right] \geq 0 .
$$

So equation (14) is satisfied with limit defined in equation (8).

In order to prove exponential tightness, there are two cases. If $\chi<0$, given $\alpha>0$, let $T_{\alpha}>\alpha / I(0)$ and define the compact set $K_{\alpha}=\left[0, T_{\alpha}\right] \cup\{+\infty\}$. Clearly

$$
\mathbb{P}\left[\tau_{u}\left(X_{n}\right) \in\left(T_{\alpha}, \infty\right)\right] \leq \mathbb{P}\left[X_{n} \in\left\{\phi: \sup _{s \in\left[T_{\alpha}, \infty\right)} \frac{\phi(s)}{1+s} \geq 0\right\}\right]
$$


That the final set is closed in $\mathcal{Y}$ can be seen by fitting an open ball around any element in its complement. Applying the functional upper bound

$$
\limsup _{n \rightarrow \infty} \frac{1}{n} \log \mathbb{P}\left[\tau_{u}\left(X_{n}\right) \in\left(T_{\alpha}, \infty\right)\right] \leq-T_{\alpha} I(0)<-\alpha .
$$

If $\chi>0$, as $I$ is strictly convex with $I(\chi)=0, x I(u / x)$ is strictly convex with $x I(u / x)=0$ if $x=u / \chi$, and therefore strictly increasing for $x>u / \chi$. For $\alpha>0$, choose $T_{\alpha}>u / \chi$ such that $J\left(T_{\alpha}\right)=T_{\alpha} I\left(u / T_{\alpha}\right)>\alpha$. Define the compact set $K_{\alpha}:=\left[0, T_{\alpha}\right]$. We have the following inequality

$$
\mathbb{P}\left[\tau_{u}\left(X_{n}\right) \in\left(T_{\alpha}, \infty\right]\right] \leq \mathbb{P}\left[X_{n} \in\left\{\phi: \sup _{t \in\left[0, T_{\alpha}\right]} \phi(t) \leq u\right\}\right] \leq \mathbb{P}\left[X_{n} \in\left\{\phi: \sup _{t \in\left[0, T_{\alpha}\right]} \frac{\phi(t)}{1+t} \leq u\right\}\right]
$$

As the last set is closed, the functional upper bound can be applied

$$
\limsup _{n \rightarrow \infty} \frac{1}{n} \log \mathbb{P}\left[\tau_{u}\left(X_{n}\right) \in\left(T_{\alpha}, \infty\right]\right] \leq-T_{\alpha} I\left(\frac{u}{T_{\alpha}}\right)<-\alpha .
$$

Thus the measures induced by $\left\{\tau_{u}\left(X_{n}\right), n \in \mathbb{N}\right\}$ are exponentially tight and satisfy the LDP with good rate-function defined in equation (8).

\section{References}

[1] A. J. Ganesh and N. O'Connell. A large deviation principle with queueing applications. Stochastics and Stochastic Reports, 73(1-2):25-35, 2002.

[2] N. O'Connell. Large deviations for the departures from a shared buffer. Journal of Applied Probability, 34:753-766, 1997.

[3] N. O'Connell. Large deviations for queue-lengths at a multi-buffered resource. Journal of Applied Probability, 35:240-245, 1998.

[4] F. Toomey. Bursty traffic and finite capacity queues. Annals of Operations Research, 79:45-62, 1998.

[5] B. M. Hambly, J. B. Martin, and N. O'Connell. Concentration results for a Brownian directed percolation problem. Stochastic Processes and their Applications, 102(2):207220,2002 .

[6] W. Whitt. Some useful functions for functional limit theorems. Mathematics of Operations Research, 5(1):67-85, 1980. 
[7] W. Whitt. Stochastic-process Limits: An Introduction to Stochastic-process Limits and Their Application to Queues. Springer Verlag, 2002.

[8] A. Puhalskii and W. Whitt. Functional large deviation principles for first-passage-time processes. Annals of Applied Probability, 7(2):362-381, 1997.

[9] R. Russell. The Large Deviations of Random Time Changes. PhD thesis, Trinity College Dublin, 1997.

[10] H. Nyrhinen. Rough descriptions of ruin for a general class of surplus processes. Advances in Applied Probability, 30:1008-1026, 1998.

[11] H. Nyrhinen. Large deviations for the time of ruin. Journal of Applied Probability, 36(1-2):733-746, 1999.

[12] A. Puhalskii. Large deviation analysis of the single server queue. Queueing Systems, 21(1-2):5-66, 1995.

[13] R. L. Dobrushin and E. Pechersky. Large deviations for random processes with independent increments on an infinite interval. Problems in Information Transmission, 4:354-382, 1999.

[14] A. Dembo and O. Zeitouni. Large Deviation Techniques and Applications. Springer, 1998.

[15] F. Riesz and B. SZ.-Nagy. Functional Analysis. Blackie and Son Limited, 1955.

[16] A. Dembo and T. Zajic. Large deviations: from empirical mean and measure to partial sums. Stochastic Processes and their Applications, 57:191-224, 1995.

[17] J-D. Deuschel and D. W. Stroock. Large Deviations. Academic Press, 1989.

[18] P. Eichelsbacher and N. O'Connell. Sample path large deviations in finer topologies. Stochastics and Stochastics Reports, 67(3-4):231-254, 1999.

[19] A. Puhalskii. Large deviation and idempotent probability. Chapman \& Hall/CRC, 2001.

[20] P. Glynn and W. Whitt. Large deviations behavior of counting processes and their inverses. Queueing Systems, 17:107-128, 1994.

[21] P. Billingsley. Convergence of Probability Measures. Wiley Inter-Science, 1999.

[22] K. Duffy and W. M. B. Dukes. On Knuth's generalization of Banach's matchbox problem. To appear in Mathematical Proceedings of the Royal Irish Academy.

[23] P. R. Halmos. Measure Theory. Van Nostrand, 1956. 